\title{
A Case Report of Airway Stenting in Tracheal Compression Secondary to Right Subclavian Artery Pseudo-aneurysm
}

\author{
Muhammad Naimmuddin Abdul Azih ${ }^{\mathrm{a}}$, Aishah Ibrahim ${ }^{\mathrm{b}}$, How Soon Hin ${ }^{\mathrm{a}}$, Kuan Yeh Chunn ${ }^{\mathrm{a}}$ \\ a Department of Internal Medicine, Hospital Tengku Ampuan Afzan, Kuantan, Pahang \\ ${ }^{\mathrm{b}}$ Respiratory Unit, Department of Internal Medicine, Hospital Tengku Ampuan Afzan, Kuantan, Pahang
}

\begin{abstract}
We report a 55-year old lady with the presentation of stridor and type II respiratory failure requiring tracheal intubation. She had right Horner's syndrome associated with pleural effusion. Her chest radiograph revealed right upper zone lobulated opacities and therefore right Pancoast tumor was the initial diagnosis. However, her CECT thorax revealed a huge right subclavian artery pseudoaneurysm with severe tracheal compression. This rare condition imposed a significant diagnostic as well as therapeutic challenge. Vascular surgery is the definitive treatment but it is associated with high risks. The exact role of rigid bronchoscopy for airway stenting is unknown due to limited evidence available. Indeed, this form of central airway obstruction may benefit from temporary tracheal stenting whilst the surgical repair of the lesion is planned. It may facilitate early weaning and allows less complicated airway control.
\end{abstract}

KEYWORDS: Tracheal compression, subclavian artery pseudoaneurysm, rigid bronchoscopy, airway stenting

\section{INTRODUCTION}

Tracheal compression is a life-threatening central airway obstruction that is commonly seen in malignant conditions. The causes can be divided into extrinsic, mural and intra-luminal lesions. Vascular aneurysm causing external tracheal compression is extremely rare. It is also a rare mimic of lung mass on chest radiograph. Indeed, CECT with CT angiogram are the investigations of choice followed by bronchoscopy to assess the severity of the tracheal narrowing with therapeutic opportunity of airway stenting.

\section{Case presentation}

Mrs LSM, a 55-year old lady, with a past history of hypertension, diabetes mellitus, obesity and recurrent stroke, presented with one week of breathlessness associated with stridor. On examination, she had right Horner's syndrome and mild pleural effusion. Her chest radiograph revealed right upper zone opacities. Her blood gas

Corresponding author:

Dr. Muhammad Naimmuddin Abdul Azih

Respiratory Unit

Department of Internal Medicine

Kulliyyah of Medicine

International Islamic University Malaysia

Kuantan, Pahang

Telephone number: 09-5704729

Email: naimedicine@hotmail.com analysis showed type II respiratory failure and she was later intubated in the intensive care unit. The initial diagnosis was right Pancoast tumor. (Figure 1)

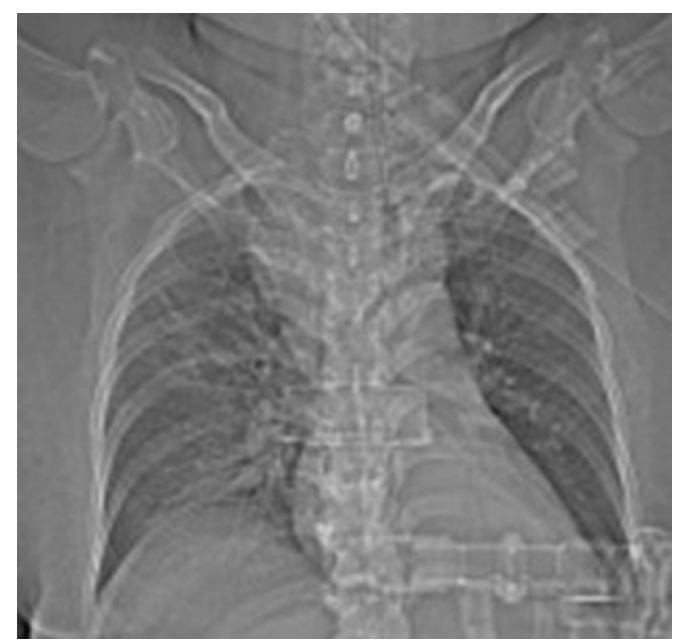

Figure 1: Chest radiograph showed a lobulated mass seen in the right upper lobe.

However, an urgent CECT thorax revealed right subclavian artery pseudoaneurysm with severe tracheal compression and carotid sheath hematoma. Further detailed reviews did not suggest a history of subclavian vein cannulation or trauma. Her connective tissue disease and infective screens were negative. (Figure 2) She was referred to vascular team and a surgical repair was planned at another tertiary referral centre. After 4 weeks of admission, her repeated CECT thorax with CT angiogram revealed a marked improvement of the tracheal 


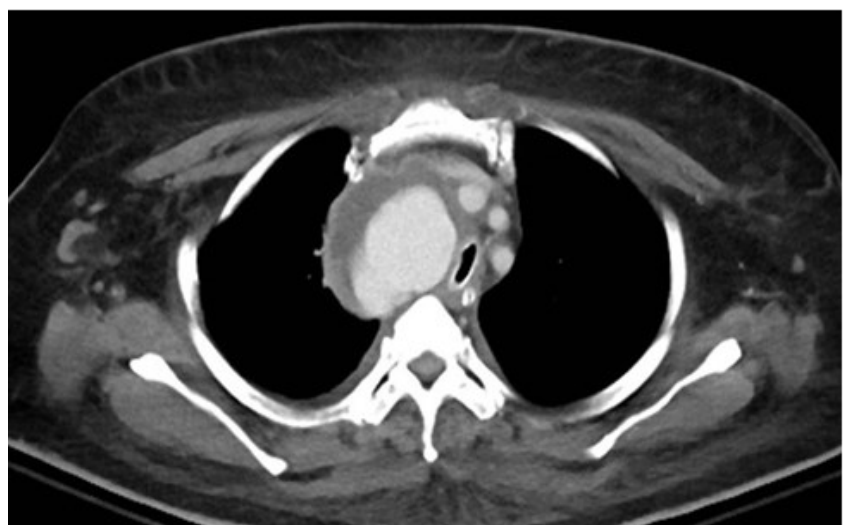

Figure 2 : CECT thorax showed a huge right subclavian artery pseudoaneurysm with marked extrinsic tracheal compression.

compression and the patient was successfully extubated by the intensive care team. She was discharged well while waiting for the date of the surgery. Unfortunately, after merely two days at home, she developed worsening breathlessness and later readmitted and intubated for severe type II respiratory failure with hospital acquired pneumonia. CT angiogram showed enlargement of the aneurysm (from $1.9 \mathrm{~cm} \times 1.9 \mathrm{~cm}$ to $5.7 \mathrm{~cm} \times 4.9 \mathrm{~cm}$ ) with worsening tracheal compression. A multidisciplinary team discussion and family meeting were conducted and it was decided that she should undergo an urgent rigid bronchoscopy with temporary airway stenting while waiting for surgical repair of the aneurysm at a distant vascular centre in order to ensure her airway patency as well as stability during transfer later. However, despite of successful Y-stent (Dumon@ silicone stent, $12 \mathrm{~mm}$ in diameter) deployment into the trachea, the airway was still externally compressed by the pressure from the arterial aneurysm (Figure 3). She eventually succumbed to the complications of stent migration and ventilator associated pneumonia.

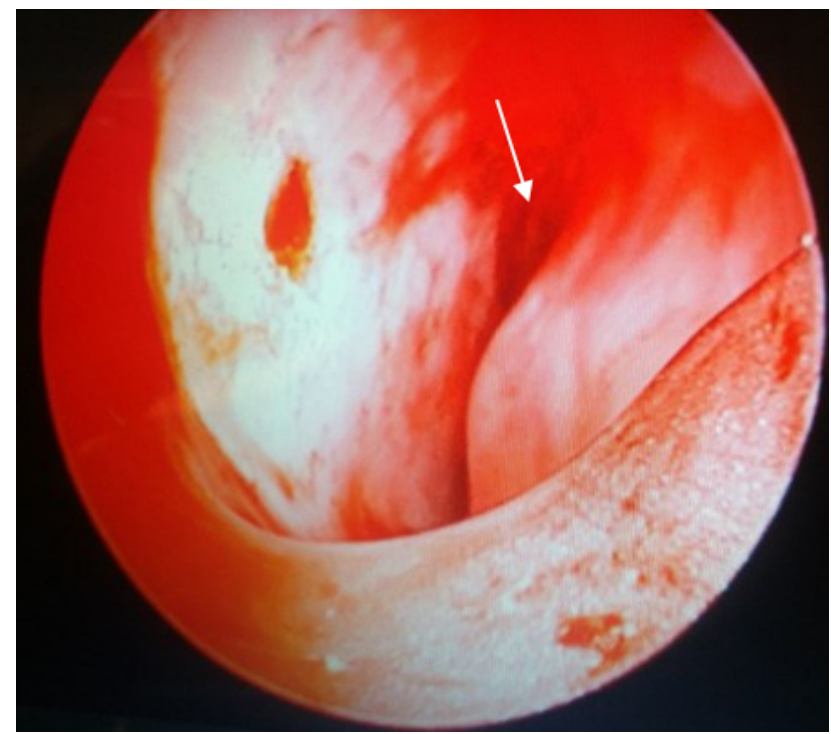

Figure 3: Rigid bronchoscopy showed a severe tracheal narrowing by an external compression. The inward bulging was pulsatile in nature (arrow).

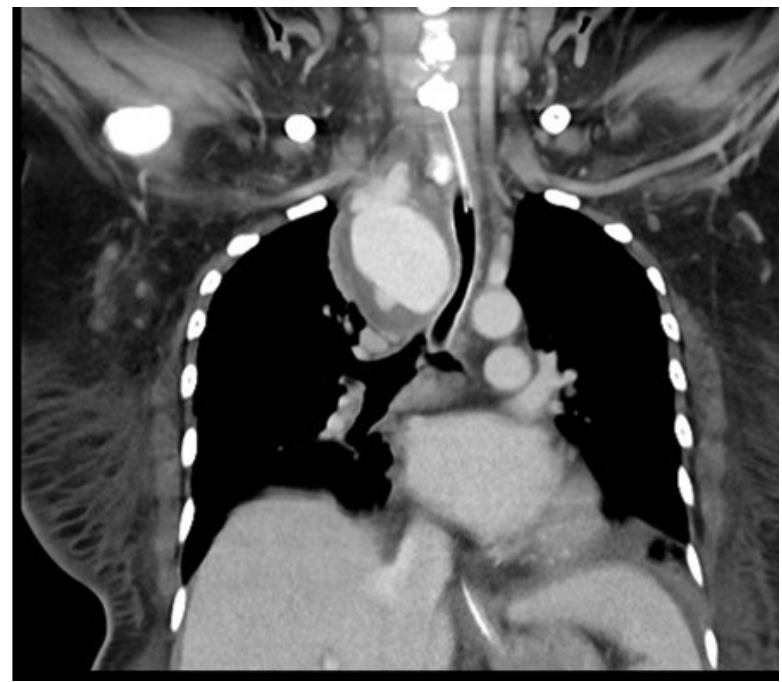

\section{DISCUSSION}

Vascular tracheobronchial compressions are relatively more common in paediatric population due to numerous congenital vascular abnormalities in this age group. In adult this condition is very rare and the exact prevalence or incidence is unknown. Apart from the more common aortic aneurysm, subclavian artery aneurysm has only been reported in a few case reports. ${ }^{1-2}$ It can be caused by atherosclerosis, trauma, infection, congenital or connective tissue diseases. The symptoms depend on the size and the location of the aneurysm itself. Extrathoracic aneurysm is commonly presented with pulsatile mass over subclavian fossa and the intrathoracic lesion tends to compress the adjacent mediastinal structures such as the trachea as seen in this case. Severe compression of the central airway may lead to respiratory failure. The definitive treatment is surgical repair. ${ }^{3}$ However, airway stenting is a feasible treatment in patients who are poor candidates for surgery or as a temporary bridging treatment in supporting the airway while waiting for the definitive surgery and it allows easier weaning from mechanical ventilation. ${ }^{4}$ Jey Chung et. al reported case series of successful weaning from mechanical ventilation by airway stenting for vascular airway compression with respiratory failure. ${ }^{5}$ Similar successful airway stentings were also reported in other case reports. ${ }^{6-7}$.

However, airway stenting imposes a great challenge due to the high risk nature of airway manipulation in patients with multiple comorbidities (e.g. advanced age, cardiorespiratory insufficiency), risk of aneurysmal rupture, airway stent related complications such as stent migration and granulation tissue formation. Additionally, the likelihood of failed stenting is also high in the case of huge aneurysm, since high pressure nature of the aneurysm can compress the stent and the resultant tracheomalacia may even worsen this effect. Even though several case reports had 
demonstrated the efficacy of silicone airway stent in vascular tracheobronchial compression syndromes, the use of metallic stent in cases of insufficient force to distend the collapsed airway by silicone stents due to forceful extrinsic vascular compression (as seen in this patient) may be deemed logical. In this case, metal airway stents have several advantages over silicone stents as its self-expanding property can generate sufficient force to distend even the firmest of strictures or compression. It is also less likely to migrate and can be inserted via flexible bronchoscopy. However, metal airway stents also have several disadvantages such as higher risk of airway perforation, more difficult to maneuver, formation of granulation tissue in between the metal struts that make removal of the stent more difficult if not impossible. ${ }^{8}$ Interestingly, the development of hybrid airway stent with metal struts (resist compression) covered by a silicone membrane (limits tumor or granulation tissue growth) may offer a good alternative . ${ }^{9}$ Indeed, head-to-head studies to compare the efficacy of metal versus silicone airway stents in vascular tracheal compression are of paramount importance to answer this clinical question.

\section{CONCLUSION}

Tracheal compression by vascular abnormalities imposes great diagnostic and therapeutic challenges. Late presentation is common due to its rarities and disingenuous clinical manifestations that mimic other conditions causing breathlessness. Bronchoscopic airway management can be a feasible option in patients with poor prognosis or as a temporary bridging treatment while the definitive treatment is planned. Whether silicone or metal airway stent should be used, more evidence is required. Silicone stent may be the initial choice, but in the cases with high risk of stent occlusion by huge high pressure compressing aneurysm, metal airway stent may provide a better airway support.

\section{REFERENCES}

1. Bicheng Zhan, Shijiang Zhang, Yongfeng shao. Operation of huge subclavian artery aneurysm: A case report. J Thorac Dis 2010; 2: 117-120.

2. M. Hohls, N. Ghanem, M. Wilhelm et al. Tracheal stenosis secondary to false aneurysm of right subclavian artery. European Journal of cardo-thoracic surgery 21 (2002) 946-947.

3. Salo JA, Ala-Kulju K, Heikkinen L et al. Diagnosis and treatment of subclavian artery aneurysms. Eur J Vasc Surg. 1990 Jun;4(3):271 -4 .

4. M. Noppen, G. Stratakos, K. Amjadi et al. Stenting allows weaning and extubation in ventilator or tracheostomy dependency secondary to benign airway
disease.Respiratory medicine (2007) 101, 139 145.

5. Jey Chung, Adnan Majid, Santacruz Jose et al. Airway Stenting for Respiratory Failure From Obstructive Vascular Anomalies: A Case Series. Chest. 2015;148.

6. David Comer, Amit Bedi, Peter Kennedy et al. Successful Endobronchial stenting for bronchial compression from a massive thoracic aortic aneurysm. J Surg Case Rep. 2010 Jun; 2010(4): 2.

7. Dutau H. Cavailles A. Fernandez-Navamuel I, Tracheal compression in a patient with Marfan's syndrome-associated tracheomegaly treated by an XXL stent: The largest diameter airway stent ever placed in a previously undescribed airway condition, Respiration 2009;77:97-101.

8. De Mello-Filho FV, Antonio SM, Carrau RL. Endoscopically placed expandable metal tracheal stents for the management of complicated tracheal stenosis. Am J Otolaryngol. 2003;24 (1):34.

9. Bolliger, CT, Arnoux, A, Oeggerli, MV, et al. Covered Wallstent insertion in a patient with conical tracheobronchial stenosis. J Bronchol. 1995; 2:215 
